\title{
Impact of COVID-19 on Gynaecological Oncology; a Global Perspective
}

\author{
Danielle O’Neill ${ }^{1}$ and Alaa El-Ghobashy ${ }^{1}$ \\ ${ }^{1}$ Royal Wolverhampton Hospitals NHS Trust
}

June 4, 2020

\begin{abstract}
The coronavirus pandemic caused global devastation and put unprecedented pressure on health care facilities world-wide. The response to the pandemic differed across the world as countries faced different challenges. Due to the novelty of this virus and its global effects, published guidance is currently limited to best practice and small-scale trials, however, globally, large-scale clinical trials are now underway. This review summarises the international response to coronavirus with respect to gynaecological oncology and suggests potential interventions to limit its spread during a resurgence or in the event of a future global pandemic.
\end{abstract}

Key Words

Coronavirus, COVID-19, gynaecological oncology, pandemic

Introduction

Following the identification of the novel coronavirus and its ensuing COVID-19 disease in late 2019, it quickly became a global pandemic. As different countries faced different challenges, new guidance was rapidly published on how to treat and control the spread of the disease. Whilst it is clear that some disparities do exist within the literature, it is evident that there are many similarities in how departments responded to this pandemic and this has been true within the field of gynaecological oncology. Whilst it is still too early for the publishing of large trials, this article aims to summarise the current available global guidance for the management of gynaecological oncology patients throughout this crisis.

Oncology patients are at risk of greater mortality as a consequence of contracting coronavirus, however do not appear to be at a higher risk of contracting the illness, with no evidence of increased incidence within this population. The increased risk of mortality is due to the immunosuppressive state of malignancy as well as current or previous administration of chemotherapeutic agents (1). A study from China has shown that the mortality of COVID-19 with a current cancer is $7.6 \%$, compared to $1.4 \%$ if no comorbidities present but was less than having comorbidities such as cardiovascular disease (13.2\%), diabetes $(9.2 \%)$, hypertension $(8.4 \%)$ and chronic respiratory disease $(8 \%)(2)$.

Although delaying surgery due to concerns about patients contracting COVID-19 peri-operatively, reduced intensive care facilities, reduced staff, reduced theatre availability and reducing risk to staff members and current patients, this needs to be offset with the risk of a rapidly progressing cancer where delay could lead to severe complications and disease progression to the point of affecting surgical cure and therefore ultimately patient prognosis.

There are several identified themes that remain common and consistent between different countries and are applicable to gynaecological oncology as a whole.

1. Reduce transmission 
2. Managing limited resources including staffing

3. Treatment prioritisation and continuation of cancer surgery

4. Exploration of alternative therapy

\section{Reduce transmission}

The main way this is achieved is through the reduction of direct contact between people. This includes through staff members and patients alike and as such, current practice required to be changed to improve the safety for everyone within the healthcare environment.

Where possible, meetings have been advised to be reduced to a minimum and performed over the internet to prevent direct contact. Within oncology, multidisciplinary team meetings are integral to the determination of oncology patients' care and as such are required to continue throughout this pandemic. Where possible, this should occur over teleconferencing or video link to reduce the risk of transmission $(3,4)$.

Outpatient activity should be reduced to limit the risk of cross-infection, particularly of high-risk patients, and alternatives such as telephone consultations or patient-initiated follow-up should be considered. Fast track referrals can be triaged and where possible postponed until the risk of COVID transmission is deemed acceptable. If postponement it not acceptable, patients should be seen in a facility where diagnostic tests, such as hysteroscopy or biopsy, can be performed during that visit to reduce attendances(5).

Women who have a confirmed diagnosis of COVID-19 should not attend for outpatient appointments(5) and should have their surgical treatment postponed for at least 15 days (3). Only once they are asymptomatic and have completed this time of isolation should their case be reviewed.

For the women whose surgery is indicated to proceed, where possible, they should be operated on in a negative-pressure theatre and this should be a dedicated theatre, free from cross-contamination. Postoperatively, patients should be transferred to dedicated wards, free from coronavirus and the route of transfer should be carefully considered to reduce cross-infection whilst transferring patients around the hospital(5).

Follow-up should be minimised and should be conducted over the telephone or online unless a recurrence is strongly suspected and additional tests should be postponed until after the pandemic except where symptoms require these to be performed or for disease monitoring (6). Additionally, post-operative follow-up for women with cervical and vulval tumours who require clinical examination which cannot be performed over telephone and as such should be seen but can be delayed by 2 months(3).

\section{Managing limited resources including staffing}

It has been estimated that approximately a third of staff may be off at any one time as a result of COVID-19 and as such surgical teams need to plan appropriately for this potential reduction in workforce(6). Reduction in elective surgical procedures has the effect of reducing the number of inpatients in both general wards and intensive care units whilst freeing theatre space for increased ITU capacity where necessary. It also allows surgical staff to be of support to those in high-intensity areas such as intensive care units and allows for flexibility within the surgical teams to allow for the potential reduction in staff. Lastly, but very importantly, it reduces the risk of cross-infection of COVID from patients admitted to the hospital for elective procedures and from the hospital to the community setting. Where surgical procedures are required to proceed, they should be performed by a senior team in order to reduce the operating time and reduce the risk of complications to help with a reduction in the length of the post-operative admission (5).

\section{Treatment prioritisation and continuation of cancer surgery}

Surgery for benign procedures should not continue in order to concentrate and direct resources to areas of greatest need (7). The pro's and con's of surgery versus pursuing alternative treatment options or delaying definitive surgery need to be clearly discussed with the patient and within the MDT. Consideration must be given to the patients' risk with aspects such as co-morbidities, age, cancer load, performance status and frailty being taken into account. Any potential for intensive care support needs to be identified as this may not be possible due to the demand secondary to COVID-19. Continuation of surgery in the presence 
of COVID-19 infection is associated with high morbidity and mortality rate, with an ITU admission of over $40 \%$ and mortality of $20 \%$ being published in the literature $(8,9)$. Patients undergoing surgery with a diagnosis of COVD-19 had post-operative respiratory complications in more than $50 \%$ of cases, which was associated with a greater mortality risk; of those that died, over $80 \%$ had respiratory complications. Cancer patients within this cohort were identified as a particularly vulnerable group with a higher risk of 30-day mortality (9). Additionally, less invasive surgical procedures such as sentinel lymph node biopsy should be considered rather than complete lymphadenectomy as the latter is associated with great morbidity and requires prolonged hospital stay, increasing the risk of COVID-19 exposure (4).

\section{Prioritisation of surgery}

Surgery within oncology is time-sensitive, a delay in receiving surgery is associated with a greater mortality due to cancer. This risk however, needs to be weighed against the risk of admission to hospital and subsequent COVID-19 exposure and the likelihood to be able to receive chemotherapy post-operatively, as per standard practice (1). Guidance has been published to help prioritise surgery for patients with gynaecological cancers to take into consideration the associated risks. It is also advised that the use of laparoscopy is avoided due to the potential risk of aerosol formation with pneumoperitoneum (10).

Emergency: operation needed within 24 hours, such as anastomotic leak, bowel perforation, torsion or rupture of ovarian cyst $(4,11)$.

Urgent: operation required within 72 hours, such as bowel obstruction or impending perforation. Operations for Gynaecological cancers should only be considered if it is considered curative or there are no other options available (4). 'Urgent' surgery should be scheduled for diagnostic procedures to enable either chemotherapy or definitive surgery to be considered $(10,11)$.

Elective: operation required within 4 weeks. The aim is for cure (4). In gynaecological oncology this would include germ cell tumours, discrete pelvic mass highly suspicious of cancer, early stage cervical cancer(12) and high-grade endometrial cancers $(4,11)$.

\section{Colposcopy}

Primary screening within the community should be withheld $(1,13,14)$. Referrals should only be made if high-grade changes on smear, borderline nuclear change in endocervical cells, possible glandular neoplasia or suspicion of invasive disease(13). In addition, women with high-grade changes must be seen within 3 months(12).

LLETZ is safe to perform as there is no evidence of viral particles in the smoke, however laser ablation and excision should be avoided due to the risk of vaporisation. Where possible, 'see and treat' procedures should be performed in one clinic attendance to reduce the number of times patients are attending the hospital(13).

\section{Exploration of alternative therapy}

Whilst the ability to perform operative interventions is restricted, MDT's need to consider the use of alternative therapies in order to manage gynaecological malignancies. Alternative therapies such as the levonorgestrel intra-uterine system have been used in patients with early endometrial cancer when surgical intervention is not possible $(4,6,15)$. These options should be considered more widely whilst the availability of definitive treatment is reduced. Where surgical intervention is possible, minimally-invasive surgery should be the gold standard (3). For intermediate or high-risk endometrial tumours, consideration should be given to vaginal hysterectomy with bilateral salpingo-oophorectomy or minimally invasive surgery with sentinel lymph node biopsy as this reduces recovery time, thus reducing patient exposure and use of resources(6).

For early ovarian tumours, minimising surgery for those women considered to be at high risk of malignancy $(\mathrm{RMI}>200)$ is suggested to remove the primary tumour and to obtain a histological diagnosis, however those women deemed to be at a lower risk of cancer $(\mathrm{RMI}<200)$ can be deferred until deemed safe to continue (15). Following this, staging can be completed with imaging or future definitive surgery with the consideration of commencing neoadjuvant therapy $(3,6)$ or prolonging current chemotherapy prior to 
definitive surgery $(3,4,6,15)$. Evidence suggests that the outcomes associated with primary surgery versus neoadjuvant chemotherapy are similar and as such presents as a viable option (16). The risks of surgery to both the patient and resources need to be considered and weighed against the risk of immunosuppression associated with chemotherapy. It has been suggested that mortality from chemotherapy is at least doubled in the presence of COVID-19 (17).

Where possible, women should be managed with spinal anaesthesia, such as in the management of cervical cancer, whereby options such as wide conisation, trachelectomy and vaginal hysterectomy can be considered depending on stage of disease. Alternatively, these patients should be managed with radiotherapy $(6,15)$. In some institutions, all women with cervical cancer are advised to be treated with chemoradiotherapy as first line(3). Equally with vulval cancer, many patients can be postponed as their lesions may be indolent, however, those patients that cannot be deferred can largely be managed with spinal anaesthesia with preference to undertake sentinel lymph node biopsy if required in order to reduce morbidity and length of admission associated with complete groin node dissection. If the tumour requires extensive surgery with reconstruction, neoadjuvant chemoradiotherapy is advised(6).

Trophoblastic tumours should be managed without delay, however low-risk women (FIGO <6) can have their methotrexate injection administered at home, whilst high-risk women are advised to continue their treatment as planned(3).

\section{Discussion}

Global pandemics, such as the coronavirus, SARS or MERS, present a unique and diverse challenge for healthcare world-wide. Whilst on one hand the treatment and care of patients with the virus is vital, this has to be balanced against the needs of patients suffering from other life-threatening illnesses. In order to achieve the optimal balance of these opposing forces, hospitals and care providers need to collaborate and pool resources in order to be as prepared as possible for the potential future impact.

This review discusses the efforts employed to reduce transmission and how limitations with resources and the need for treatment prioritisation can be managed in order to allow those women with gynaecological cancers access to the care they require and deserve. It also highlights where we can afford to offer additional therapies in place of surgical management where the risks to the patient and public health are perceived too great.

Consideration must be given to planning for the months following the peak of the pandemic as there is inevitably a backlog of patients requiring urgent surgery who will need to be prioritised as soon as practicable. For all women going through the cancer pathway it is an extremely stressful time, however having to go through it during this time of uncertainty and fear is likely to cause significant emotional distress and this needs to be appreciated by the attending healthcare professionals and support provided to the patient and their families where possible. The importance of keeping patients updated with their care and involved in the discussions surrounding their treatment cannot be understated.

As a healthcare organisation, the NHS has been pushed to its limits to effectively manage the effects of the coronavirus pandemic. All guidance we have to date is reliant on small studies and expert advice and further research is required to provide evidenced-based guidance to improve preparedness for any future resurgence or alternative pandemic.

\section{Current research applicable to gynaecological oncology}

In the current climate, all ongoing trials within the field of gynaecological oncology have been suspended. Within the UK, NHS in collaboration with Universities are focusing their resources on coronavirus-related trials.

There are many research trials underway globally investigating how coronavirus has impacted services involved with gynaecological oncology. Although still recruiting, these will be an important learning resource 
for future guidance and to tailor our current guidance to align with scientifically conducted research. Table 1 briefly describes each of these current ongoing studies.

\section{Preparing for future pandemic}

In order to develop our healthcare service, it is vital that we learn from this experience to improve our preparedness for future pandemic situations. The lessons main learned are those surrounding management of resources in order to appropriately manage the pandemic situation, whilst maintaining sufficient services to allow the management of other patients with time-critical illness. Firstly, organisation of staffing and preparing for staff sickness and absence is vital in order to maintain the service. To achieve this, prompt cessation of elective theatre and clinics should be instated to enable staff to be redeployed to areas of greatest need and unavoidable surgery needs to be prioritised appropriately to limit patient throughflow. Any required meetings should be performed virtually to reduce risk of transmission. Patients should be manged through virtual clinics where possible and if patient attendance is absolutely necessary, all investigations should be performed during the same visit and treatment commenced where possible to limit patient appointments. Routine screening should be postponed until after the pandemic. Non-infective patients should be managed in a geographically distinct area from those with infection and surgery should be performed in a negative pressure environment to limit transmission. In addition, surgical procedures may need to be altered to reduce operating time, reduce post-operative recovery and its associated hospital admission. Alternatives to surgery should be explored where possible to reduce the number of patients requiring admission where they risk exposure to infection and occupy limited resources. Finally, planning needs to be commenced early regarding the reinstating of services in order to deal with the incurred backlog secondary to the pandemic to ensure patients are followed up appropriately to avoid excess morbidity and mortality.

\section{Conclusion}

The COVID-19 pandemic has put unprecedented pressure on health care systems globally and the consequence of this to cancer patients is yet to be determined. Gynaecological oncology services are required to manage their patients in a sensitive and empathetic manner, acknowledging how stressful it is to undergo oncology treatment during such disruption. Services and protocols should be regularly audited and amended to keep pace with changing demands in order to ensure the best care is provided to all patients. Finally, individual institutions and countries are required to collaborate in order share information and services to advance knowledge and treatment during such times of stress for the greater good of the population.

\section{Disclosure of interests}

There are no disclosure of interests.

\section{Contribution of authorship}

AEG was involved in the conception of the paper, DO and AEG wrote, edited and agreed on the final version of the article.

Ethics approval

\section{NA}

\section{Funding}

No funding was received for this article.

\section{References}


1. Bhatla N, Singhal S. The COVID-19 Pandemic and Implications for Gynaecologic Cancer Care. Indian Journal of Gynecologic Oncology. 2020.

2. Aylward, Bruce (WHO); Liang W (PRC). Report of the WHO-China Joint Mission on Coronavirus Disease 2019 (COVID-19). WHO-China Jt Mission Coronavirus Dis 2019 [Internet]. 2020;2019(February):16-24. Available from: https://www.who.int/docs/default-source/coronaviruse/who-china-joint-mission-on-covid19-final-report.pdf

3. Akladios C, Azais H, Ballester M, Bendifallah S, Bolze P-A, Bourdel N, et al. Recommendations for the surgical management of gynecological cancers during the COVID-19 pandemic - FRANCOGYN group for the CNGOF. J Gynecol Obstet Hum Reprod. 2020;(2019):101729.

4. Society BGC, Gynaecologists RC of O and. Framework for care of patients with gynaecological cancer during the COVID-19 pandemic. 2020;(March).

5. COVIDSurg Collaborative. Global guidance for surgical care during the COVID-19 pandemic. Br J Surg [Internet]. 2020;(March). Available from: http://www.ncbi.nlm.nih.gov/pubmed/32293715

6. S.E.G.O SS of O and G. GYNECOLOGY AND OBSTETRICS Recommendations of the Gynecological Oncology and Breast Disease Section of SEGO on management of gynecological tumors during the COVID-19 pandemic General measures Endometrial cancer. :1-4.

7. European Society for Gynaecological Endoscopy. ESGE Recommendations on Gynaecological Laparoscopic Surgery during Covid-19 Outbreak. Available from: https://esge.org/wpcontent/uploads/2020/03/Covid19StatementESGE.pdf

8. Lei S, Jiang F, Su W, Chen C, Chen J, Mei W, et al. Clinical characteristics and outcomes of patients undergoing surgeries during the incubation period of COVID-19 infection. EClinicalMedicine. 2020;

9. CovidSurg Collaborative. Mortality and pulmonary complications in patients undergoing surgery with perioperative SARS-CoV-2. Lancet. 2020;(In press)(20):1-12.

10. Ramirez PT, Chiva L, Eriksson AGZ, Frumovitz M, Fagotti A, Gonzalez Martin A, et al. COVID-19 Global Pandemic: Options for Management of Gynecologic Cancers. Int J Gynecol Cancer. 2020;ijgc-2020001419.

11. Oncology ES of M. ESMO MANAGEMENT AND TREATMENT ADAPTED RECOMMENDATIONS IN THE COVID-19 ERA: EPITHELIAL OVARIAN CANCER [Internet]. 2020 [cited 2020 Apr 24]. Available from: https://www.esmo.org/guidelines/cancer-patient-management-during-the-covid-19pandemic/gynaecological-malignancies-epithelial-ovarian-cancer-in-the-covid-19-era

12. Pathology AS for C and C. ASCCP Interim Guidance for Timing of Diagnostic and Treatment Procedures for Patients with Abnormal Cervical Screening Tests [Internet]. [cited 2020 Apr 24]. Available from: https://www.asccp.org/covid-19

13. Pathology BS for C and C. Colposcopy guidance during COVID 19 pandemic [Internet]. Available from: https://www.bsccp.org.uk/assets/file/uploads/resources/Colposcopy_guidance_COVID_19_pandemic.V3.pdf

14. Oncology AS of C. COVID-19 Patient Care Information [Internet]. [cited 2020 Apr 24]. Available from: https://www.asco.org/asco-coronavirus-information/care-individuals-cancer-during-covid-19

15. Obstetrics IF of G and. COVID-19 \& Management of Gynecological Cancers [Internet]. [cited 2020 Apr 24]. Available from: https://www.figo.org/covid-19-management-gynecological-cancers

16. Vergote I, Coens C, Nankivell M, Kristensen GB, Parmar MKB, Ehlen T, et al. Neoadjuvant chemotherapy versus debulking surgery in advanced tubo-ovarian cancers: pooled analysis of individual patient data from the EORTC 55971 and CHORUS trials. Lancet Oncol. 2018; 
17. Williams M, Le Calvez K, Mi E, Chen J, Dadhania S, Pakzad-Shahabi L. Estimating the Risks from COVID-19 Infection in Adult Chemotherapy Patients. 2020;2. Available from: http://dx.doi.org/10.1101/2020.03.18.20038067\%0Ahttps://www.medrxiv.org/content/medrxiv/early/2020/03/20/2020.03.1

\section{Hosted file}

Table of trials.docx available at https://authorea.com/users/329668/articles/456614-impactof-covid-19-on-gynaecological-oncology-a-global-perspective 